\title{
Prevention of Femoral Head Deformity after Ischemic Necrosis Using Ibandronate Acid and Growth Factor in Immature Pigs
}

\author{
Bibiana Dello Russo, Eduardo Luis Baroni, Nicolas Saravia, Valeria Amelong, Fabiana Lubiniecky, \\ Marcelo Asprea, Gustavo Williams, Susana Rodriguez \\ J. P. Garrahan Hospital Nacional de Pediatria Ciudad Autónoma de Buenos Aires, Buenos Aires, Argentina \\ Email: bibianadellorusso@yahoo.com.ar
}

Received December 31, 2011; revised February 28, 2012; accepted March 9, 2012

\begin{abstract}
Background: Femoral head deformity is the most severe sequela of ischemic necrosis in skeletally immature patients. Development of severe deformity shortens useful survival time of the joint due to the appearance of early degenerative changes. Preservation of the trabecular architecture through inhibition of osteoclastic bone resorption may minimize the development of the deformity in an animal model of ischemic necrosis of the femoral head. Aims: To determine if a highly potent antiabsorptive agent, ibandronate, would inhibit bone resorption during necrotic femoral head repair to avoid subsequent flattening and deformity, to determine if the use of platelet-rich plasma stimulates bone repair and neovascularization of the damaged femoral head, and to evaluate if the combination of both therapies can preserve the femoral head while stimulating new bone formation in an animal model of ischemic necrosis. Methods: Ischemic necrosis of the femoral head was induced by surgical ligature of the circumflex vessels in 10 Landrace pigs. The animals were divided into four different groups and were administered ibandronate acid, platelet-rich plasma, or both. The contralateral, untreated femoral heads with surgical ligature of the circumflex vessels served as the control group. All animals were killed three months after surgery and the femoral head was evaluated both radiographically and histologically. The femur length was measured on radiographs and compared among the groups. Results: Final femoral length was significantly longer in the group treated with a combination of both therapies (platelet-rich plasmaibandronate acid) compared to the others groups, with a significant difference between groups. The histological findings showed increased osteoblastic activity and thickened trabiculae, a higher rate of neovascularization, and focal hyperplasia greater bone resorption and neovascularization. Only slight changes (femoral length) were observed in the animals that received platelet-rich plasma in situ favoring revascularization that was, however, only seen in the first months of administration. Conclusions: Radiographic and histological studies showed that a combination of both therapies (platelet-rich plasma and ibandronate acid) preserved the trabecular architecture and prevented femoral head deformity in the early phase of ischemic necrosis repair in immature pigs, coinciding with reports by other authors. Clinical Relevance: These findings support the concept that a combination of antiresorptive and anabolic agents can significantly improve bone healing and decrease femoral head deformity following ischemic necrosis in the fragmentation stage. Further studies would be necessary to determine the optimal dose and longterm effectiveness for the use in pediatric patients.
\end{abstract}

Keywords: Perthe’s Disease; Avascular Necrosis; Ibandronate Acid; Platelet-Rich Plasma

\section{Introduction}

Femoral head deformity is the most severe sequela of ischemic necrosis in skeletally immature patients. Development of severe deformity shortens useful survival time of the joint due to the appearance of early degenerative changes.

Reossification and cure occur subsequently, but residual deformity of the femoral head persists [1-3]. The onset of the deformity is associated with the process of necrosis repair. In this process, resorption of the necrotic bone by osteoclasts results in mechanical compromise of the femoral head leading to flattening of the structure of the epiphysis. The mechanical weakening of the femoral head plays an important role in the pathogenesis of the deformty,caused by pathological bone remodeling with predominance of bone resorption without subsequent coupled bone formation (imbalance between catabolism and anabolism). 
We hypothesized that preservation of the trabecular architecture through inhibition of osteoclastic bone resorption may minimize the development of the deformity in an animal model of ischemic necrosis of the femoral head. Based on this hypothesis, the following study aims were proposed:

1) To determine if a highly potent antiabsorptive agent —ibandronate — would inhibit bone resorption during necrotic femoral head repair to avoid subsequent flattening and deformity.

2) To determine if the use of local administration of platelet-rich plasma would stimulate bone repair and neovascularization of the damaged femoral head [4].

3) To determine if the combination of both therapies can preserve the femoral head while stimulating new bone formation in an animal model of ischemic necrosis.

\section{Material and Methods}

The study was conducted according to the parameters established in the protocols of anesthesia and analgesics of the Animal Protection Society, which were used in all treatment phases. The investigation protocol was approved by the Committee of Research and Development at our institution. For this experimental model, 10 3-month-old Landrace pigs weighing between 10 and $15 \mathrm{~kg}$ were used.

Ischemic necrosis of the femoral head was induced by a surgical ligature placed tightly around the femoral neck and transecting the ligamentum teres in 10 pigs weighing between 10 and $15 \mathrm{~kg}$ [5]. Subsequently, ibandronate acid and/or platelet-rich plasma were administered according to the groups [4].

Radiographic and pathological studies were performed. The animals were divided into two groups and two subgroups each:

GROUP A: Consisting of five pigs who received intravenous ibandronate acid.

The right hip (subgroup A1) was then intraarticular injected with platelet-rich plasma extracted from the blood of the pig itself, while in the left hip (subgroup A2) no subsequent procedure was performed.

GROUP B: Consisting of five pigs that did not receive ibandronate acid.

Similar to group A, the right hip (subgroup B1) was intraarticular injected with platelet-rich plasma, while in the left hip (subgroup B2) no procedure was performed.

Surgical Technique: Under general anesthesia and in the lateral decubitus position, the hip was exposed through a longitudinal approach. After incision in the skin, celular tissue, and fascia and separation of muscle mass, capsulotomy and transection of the ligamentum teres were performed (see Figure 1).

After ligature of the circumflex vessels at the level of the femoral neck with nonreabsorbable sutures, the wound was closed in multi-layers. The animals were allowed to bear weight immediately aftersurgery (see Figure 2).

From the tenth day after surgery onward, the animals in group A received injections of ibandronate acid at a dose of 44 micrograms per kilogram per day subcutaneously, three times a week for six weeks.

Platelet-rich plasma were injected in the right hip (subgroups A1 and B1) of the animals in both groups. It was obtained using the following procedure: $5 \mathrm{ml}$ of blood was drawn, placed in special anticoagulant-coated tubes, and centrifuged at $3000 \mathrm{rpm}$ for 10 minutes. The supernatants were then removed under a laminar flow hood. The samples were placed in sterile tubes and again centrifuged at $2000 \mathrm{rpm}$ for 15 minutes at room temperature. The supernatants were again removed under the laminar flow hood until reaching the platelet pellet. An aliquot was taken to determine the platelet count. These tubes were sent to the surgery room with $10 \%$ calcium chloride as a precipitant [4] (see Figure 3).

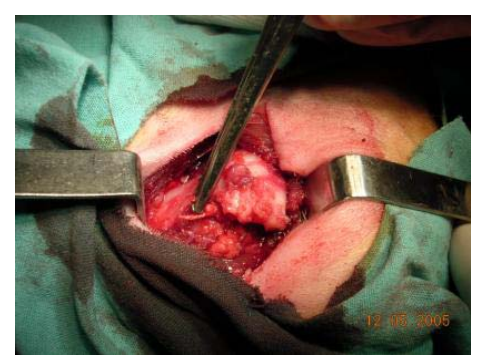

Figure 1. Identification of the joint, capsulotomy, and transection of the ligamentum teres.

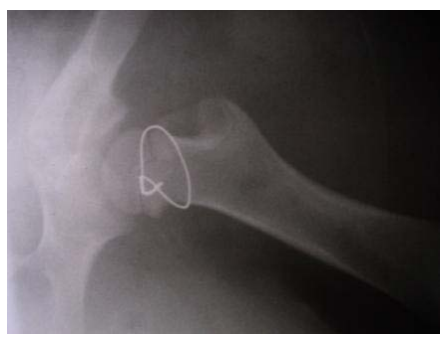

Figure 2. Ligature of the circumflex vessels at the level of the femoral neck. In this specimen, the procedure was performed with a wire loop to radiologically confirm adequate position of the ligature.

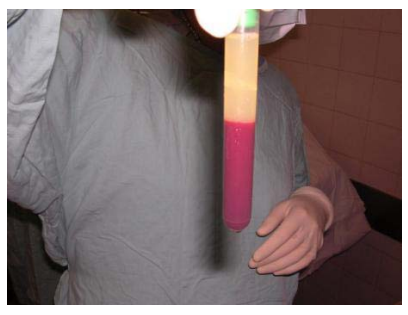

Figure $3.5 \mathrm{ml}$ was placed in anticoagulant-coated tubes and centrifuged. From the supernatant, the platelet pellet was obtained. 
The following anesthetic protocol was used: premedication with atropine sulfate at $1 \mathrm{mg} / \mathrm{kg}$, a $30 \mathrm{mg} / \mathrm{kg}$ dose of ketamine, and midazolan at $0.1 \mathrm{mg} / \mathrm{kg}$, via the intramuscular route. Following inhalant premedication, general anesthesia was induced with isoflurane (5\%) and then maintained $0.5 \%$ with the aggregate of a 1 gama $/ \mathrm{kg}$ intravenous dose of fentanyl. Antibiotic prophylaxis with intramuscular enrofloxacin at $0.1 \mathrm{mg} / \mathrm{kg}$ was used. All animals were killed three months after surgery. The necrotic femoral heads were evaluated with anteroposterior X-rays and pathological study. Radiographic control studies were performed presurgically, immediately after surgery, three months after surgery, and after the pigs were sacrificed. The epiphyseal quotient was calculated dividing maximum epiphysis height by maximum physeal-plate diameter on simple anteroposterior X-rays and final femur length (see Figure 4).

The femoral heads were sent for pathological evaluation. Section was performed at the level of the central weight-bearing region. Following decalcification in EDTA, 4-mm-thick slices were fixed and stained with hematoxylin and eosin.

Macro- and micro changes were assessed based on the following parameters: state of the joint cartilage, physis, metaphysis, presence of inflammatory changes, and secondary ossifications (see Figure 5).

\section{Statistical Analysis}

For statistical analysis, a data registration list was made in Excel. Descriptive results are reported as mean, median, and standard deviation. Epiphyseal quotient (EQ), femur length, and macrochanges of the femoral heads were evaluated in each gropus. For comparative analysis we

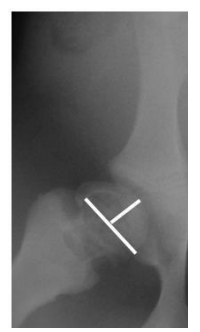

Figure 4. Measurement of the epiphyseal quotient (EQ).

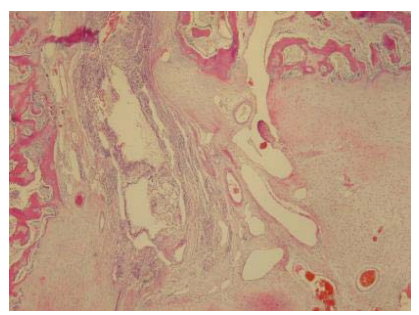

Figure 5. Histological section showing changes in vascularization, fibrosis, and a nucleus of secondary ossification. used ANOVA test for independent samples with Bonferroni correction; and Bartlett's test was used for variances evaluation between groups. All the analyses and graphics were carried out with STATA 9.1. (StataCorp. Texas).

\section{Results}

\subsection{Complications}

Two animals died in the postsurgical period. One pig from group A died 6 weeks postoperatively due to sepsis of the hip and one pig from group B died immediately postoperatively because of atypical pneumonia causing acute respiratory failure. One pig had a postoperative hematoma in the femur, which resolved without treatment.

\subsection{Pathological Findings}

All of the surviving pigs (8 animals) developed necrosis of the epiphysis.Necrotic changes in the bone marrow and empty trabecular lacunae were observed.The hips of the animals in group A (Subgroup A1 and A2) showed increased osteoblastic activity and thickened trabiculae, a higher rate of neovascularization, and focal hyperplasia. Isolated nuclei of secondary ossification were observed in three of these animals (see Figures 6 and 7).

In the hips that were not treated with ibandronate (Group B), areas with increased bone resorption, fibrosis, and loss of trabecular architecture with irregularities in the bone distribution were found.

Macroscopically, the femoral heads showed marked

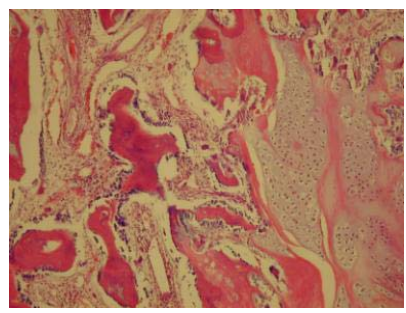

Figure 6. Histological specimen of a hip from group A showing increased osteoblastic activity and a higher rate of neovascularization.

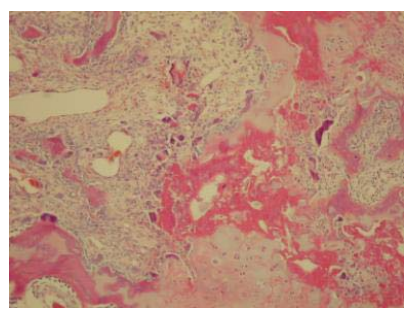

Figure 7. Histological specimen of a hip from group B showing loss of trabicular organization and increased bone resorption. 
differences between groups. They were categorized according to the following criteria:

1) Conserved joint surface and sphericity.

2) Conserved sphericity with alteration of the joint surface.

3) Alteration of sphericity and joint surface.

4) Marked deformity of the joint structure.

\subsection{Radiological Findings}

Final length of the femur was significantly longer in the group treated with a combination of both therapies (group A1) and ibandronate alone (group A2) compared to the group that did not receive the drug (group B1: with platelet rich plasma and group B2) These differences were significant statistically ( $p$ value $<0.001$ ) (see Table 1 and Graphic 1).

The epiphyseal quotient was similar in both groups $\mathrm{A}$ (with medication) $(p<0.001)$ and higher than in groups $B$ without medication. However, no difference was found between subgroups A at examination four month post-

Table 1. Femur length by groups.

\begin{tabular}{ccc}
\hline Group & Femur length $(\mathrm{mm})$ Mean $( \pm$ SD $)$ & number \\
\hline B2 & $10.55( \pm 0.24)$ & 4 \\
B1 & $11.5( \pm 0.22)$ & 4 \\
A2 & $12.27( \pm 0.17)$ & 4 \\
A1 & $12.32( \pm 0.17)$ & 4 \\
Total & $11.66( \pm 0.76)$ & 16 \\
\hline
\end{tabular}

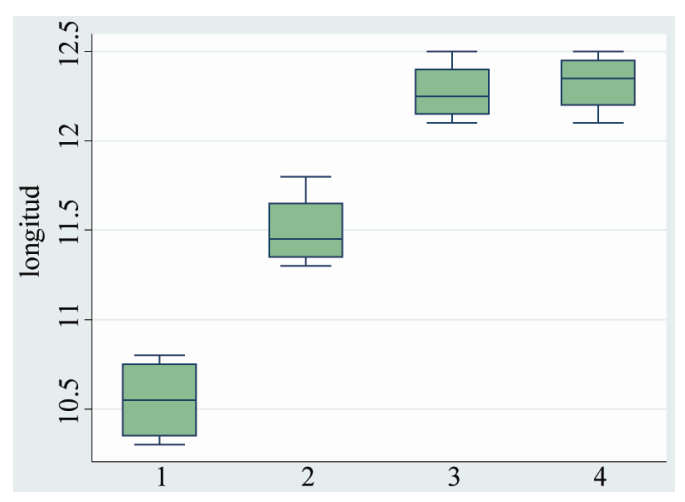

Graphic 1. Femur length by groups.

Table 2. Epiphyseal quotient (4 month post-surgery).

\begin{tabular}{ccc}
\hline Group & Epiphyseal quotient Mean $( \pm$ SD $)$ & Freq. \\
\hline B2 & $0.56( \pm 0.22)$ & 4 \\
B1 & $0.58( \pm 0.19)$ & 4 \\
A2 & $0.64( \pm 0.13)$ & 4 \\
A1 & $0.63( \pm 0.09)$ & 4 \\
Total & $0.60( \pm 0.04)$ & 16 \\
\hline
\end{tabular}

surgery (see Table 2 and Graphic 2).

The epiphyseal quotient was 0.63 greater in group A (both subgroups with medication) than in group B without medication (both subgroups): 0.40. ( $<<0.0001$ ). There were no significant differences with the subgroups treated with platelet-derived growth factors (subgroups A1 and B1).

All animals that were treated with ibandronate presented with radiodense bands near the growth-plate cartilage (see Table 3 and Graphic 3).

\section{Discussion}

Residual femoral head deformity is the most severe sequela of ischemic necrosis of the immature femoral head as it may seriously affect joint survival.

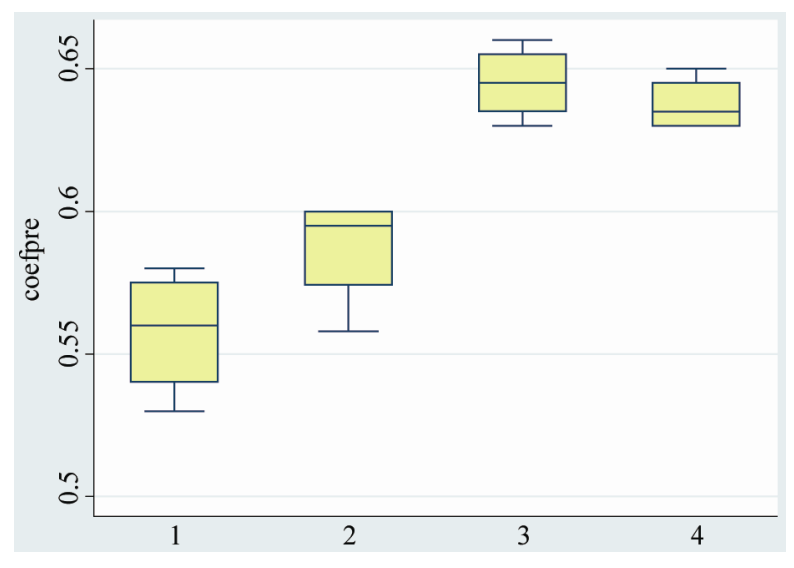

Graphic 2. Epiphyseal quotient (4 month post-surgery).

Table 3. Epiphyseal quotient (end of the study).

\begin{tabular}{ccc}
\hline Group & Epiphyseal quotient Mean $( \pm$ SD) & Freq. \\
\hline B2 & $0.53( \pm 0.012)$ & 4 \\
B1 & $0.57( \pm 0.012)$ & 4 \\
A2 & $0.63( \pm 0.012)$ & 4 \\
A1 & $0.66( \pm 0.012)$ & 4 \\
Total & $0.60( \pm 0.05)$ & 16 \\
\hline
\end{tabular}

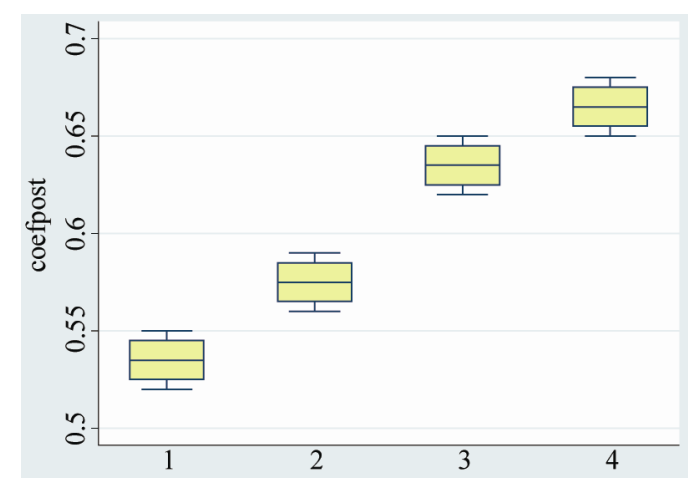

Graphic 3. Epiphyseal quotient (end of the study). 
The repair process following ischemic necrosis contributes to the development of the deformity. Loss of trabecular architecture in the early phase of the repair compromises the structural integrity of the femoral head with flattening of the osseous epiphysis during weight bearing. Finally, the bone heals with residual deformity [6].

There is current evidence that mechanical weakening of the femoral head and its components, bone and cartilage, occurs after ischemic necrosis in the piglet model [7]. The mechanical properties of the infracted femoral heads are affected. Histopathological studies of femoral head samples from patients with Legg-Calvé-Perthes disease show areas of bone resorption with a lack of new bone formation [8]. In these kind of patients changes in bone resorption occur in the fragmentation stage followed by a repair process consisting of bone-growth stimulation and bone apposition [9,10]. Effective treatment of ischemic necrosis would consist of impeding bone resorption thus avoiding collapse and deformity. Biphosphonates inhibit resorption of necrotic bone by osteoclast-mediated bone resorption and improve the preservation of the infarcted femoral head. This mechanism may contribute to reduce femoral head deformation and preserve sphericity maintaining the structure of the femoral head even in a rapidly growing animal model. Based on this hypothesis, high doses of ibandronate were used in the present study (optimal dose for an adult ovariectomized animal: $10 \mathrm{microgram} / \mathrm{kg} / \mathrm{d}$ ) [11]. Ibandronate acid is a biphosphonate that inhibits osteoclastic bone resorption and has proven to be fifty times more potent than pamidronate and ten times more potent than alendronate [2]. Pamidronate and alendronate did not alter the growth rates of children with osteogenesis imperfecta and osteoporosis secondary to diffuse connective-tissue diseases.

In an animal model in rats with surgically induced osteonecrosis, zolendronic acid treatment resulted in a significant increase in trabecular number and improved femoral shape too. Prophylactic zolendronic acid almost completely preserved the structure of the femoral head $[12,13]$.

In another study in rats with Perthes-like osteonecrosis, zolendronic-acid treatment resulted in significant improvement of the epiphyseal quotient and the number of animals with "flat" femoral heads was reduced in treated groups compared with controls [5]. Preclinical studies are necessary to determine the effect in children population .

In our study, Ibandronate seems to become effective when revascularization of the necrotic areas initiates, around three weeks after the ischemic event. Thus, results were already observed in the first month in the two groups that received the drug, however, the results in these groups intensified and became more marked after three months of use. Platelet-rich plasma has the potential to affect cartilage biology, and has been shown to increase DNA content as well as proteoglycan and collagen synthesis in chondrocytes, had numerous important limitations at this time due to variability between various commercial PRP systems and Variability in:

- Platelet recovery

- Growth factor content per platelet

- Inclusion/exclusion of WBCs

- Fibrin content

— Timing of platelet activation

- Kinetics of cytokine release from the PRP [14]. In this study after the fourth month, a marked difference was found between the group that received platelet-rich plasma and those that did not, suggesting a stimulating effect on neovascularization. This effect was afterwards concealed by the effect of ibandronate.

\section{Conclusion}

Radiographic and histological studies showed that ibandronate acid preserved the trabecular architecture and prevented femoral head deformity in the early phase of ischemic necrosis repair in immature pigs, coinciding with reports by other authors [3]. Further studies would be necessary to determine the optimal dose and longterm effectiveness for the use in pediatric patients. Only slight changes (femoral length) were observed in the animals that received platelet-rich plasma in situ. These factors favored revascularization that was seen only in the first months of administration.

\section{Significance}

These findings support the concept that a combination of antiresorptive and anabolic agents can significantly improve bone healing and decrease femoral head deformity following ischemic necrosis in fragmentation period of the Perthes disease.

\section{REFERENCES}

[1] H. K. W. Kim, P.-H. Su and Y.-S. Qiu, "Histopathologic Changes in Growth-Plate Cartilage Following Ischemic Necrosis of the Capital Femoral Epiphysis: An Experimental Investigation in Immature Pigs,” Journal of Bone \& Joint Surgery, Vol. 83A, No. 5, 2001, pp. 688-697.

[2] H. K. W. Kim and P.-H. Su, "Development of Flattening and Apparent Fragmentation Following Ischemic Necrosis of the Capital Femoral Epiphysis in a Piglet Model,” Journal of Bone \& Joint Surgery, Vol. 84, No. 8, 2002, pp. 1329-1334.

[3] H. K. W. Kim, T. S. Randall, H. Bian, J. Jenkins, A. Garces and F. Bauss, "Ibandronate for Prevention of Femoral Head Deformity after Ischemic Necrosis of the Capital Femoral Epiphysis in Immature Pigs,” Journal of 
Bone \& Joint Surgery, Vol. 87, No. 3, 2005, pp. 550-557. doi:10.2106/JBJS.D.02192

[4] J. R. Lieberman, A. Daluiski and T. A. Einhorn, "The Role of Growth Factors in the Repair of Bone. Biology and Clinical Application,” Journal of Bone \& Joint Surgery, Vol. 84, No. 6, 2002, pp. 1032-1034.

[5] J. E. Kenzora, R. E. Steele, Z. H. Yosipovitch and M. J. Glimcher, "Experimental Osteonecrosis of the Femoral Head in Adult Rabbits," Clinical Orthopaedics, Vol. 130, 1978, pp. 8-46.

[6] A. Barquet, "Natural History of Avascular Necrosis Following Traumatic Hip Dislocation in Childhood: A Review of 145 Cases,” Acta Orthopaedica Scandinavica, Vol. 53, 1982, pp. 815-820. doi:10.3109/17453678208992299

[7] D. Pringle, T. Koob and H. Kim, "Indentation Properties of the Growing Femoral Head Deformity Following Ischemic Necrosis," Journal of Orthopaedic Research, Vol. 22, No. 1, 2004, pp. 122-130. doi:10.1016/S0736-0266(03)00135-9

[8] A. Catterall, et al., "A Review of the Morphology of Perthes' Disease,” Journal of Bone \& Joint Surgery, British Volume, Vol. 64B, No. 3, 1982, pp. 269-275.

[9] S. L. Weinstein, “Legg-Calvé-Perthes Syndrome,” In: R. T. Morrisy and S. L. Weistein, Eds., Lovell and Winter's
Pediatrics Orthopaedics, 5th Edition, Lippincott Williams and Wilkins, Philadelphia, 2001, pp. 957-998.

[10] S. D. Stulberg, D. R. Cooperman and R. Wallensten, "The Natural History of Legg-Calvé-Perthes Disease," Journal of Bone and Surgery, Vol. 63, 1981, pp. 10951098.

[11] F. Bauss, M. Wagner and L. H. Hothorn, "Total Administered Dose of Ibandronate Determines Its Effects on Bone Mass and Architecture in Ovatriectomized Aged Rats,” Journal of Rheumatology, Vol. 29, No. 5, 2002, pp. 990-998.

[12] D. Little, et al., "Zolendronic Acid Treatment Results in Retention of Femoral Head Structure after Traumatic Osteonecrosis in Young Winstar Rats," Journal of Bone Mineral Research, Vol. 18, No. 11, 2003, pp. 2016-2022. doi:10.1359/jbmr.2003.18.11.2016

[13] D. Little, et al., "Zolendronic Acid Improves Femoral Head Sphericity in a Rat Model of Perthes Disease," Journal of Orthopaedic Research, Vol. 23, No. 4, 2005, pp. 862-868. doi:10.1016/j.orthres.2004.11.015

[14] L. Y. Carreon, et al., "Platelet Gel (AGF) Fails to Increase Fusion Rates in Instrumented Posterolateral Fusions,” Spine, Vol. 30, No. 9, 2005, pp. E243-E246. doi:10.1097/01.brs.0000160846.85397.44 